\title{
INVESTIGACIONES
}

\section{Formador de la educación superior técnico profesional: Percepciones sobre su trabajo docente}

\author{
Trainers of technical professional higher education: Perceptions of their teaching
}

\author{
Formador da educação superior técnica profissional: \\ Percepções sobre seu trabalho docente
}

\begin{abstract}
Carolina Barrientos Delgado, Antonio Navío Gámez. ${ }^{a}$
${ }^{a}$ Grupo de Investigación CIFO, Departamento de Pedagogía Aplicada, Universidad Autónoma de Barcelona, Bellatera, España. Fono: 34935812679. Correos electrónicos: carolinapaz.barrientos@e-campus.uab.cat, antoni.navio@uab.cat
\end{abstract}

\section{RESUMEN}

El estudio exploró y analizó las percepciones que tienen los profesionales de la Educación Superior Técnico Profesional chilena que trabajan en la industria y realizan docencia como parte de su jornada laboral. Se describió cómo sus aspectos personales, formación académica, experiencia profesional y los recursos del entorno afectan en su rol docente, y qué importancia conceden a sus funciones de planificación, desarrollo y evaluación de la formación. Se evidenció que su labor se basa en modelos que traen de sus preconcepciones docentes o de la relación con sus pares. Su alto grado de especialización técnica, traducida en formación, es un elemento de motivación para realizar docencia. Se discute que ser un buen profesor con dedicación parcial e identificarse con ello tiene algo de logro personal, pero también es una consecuencia del modo de operar, gracias al apoyo de un grupo de trabajo, un departamento, procedimientos, valores y formación permanente.

Palabras clave: educación superior técnico profesional, formador de la educación superior técnica profesional, rol docente.

\section{ABSTRACT}

This study explored and analyzed the perceptions of technical training professionals. We centered on teachers of technical professional higher education in Chile who work for the industry and who teach in a part-time schedule. We described how their personal history, education, training, professional experience and resources affect their professional performance as teachers, as well as, the functions of planning, development and assessment of their teaching. The results showed that their tasks reflect models created from relationships with peers or from models that they bring from their preconceptions. A high level of technical expertise, which results in the training, is an element of motivation when teaching. We discuss the idea that while a good part-time teacher is somewhat of a personal achievement; it is also a result of operating model thanks provided by working groups, values, teaching methods, and constant learning.

Key words: technical professional higher education, trainer of the technical professional higher education, teaching role.

\section{RESUMO}

O estudo explorou e analisou as percepções que possuem os profissionais da Educação Superior Técnica Profissional chilena que trabalham em indústrias e que dão aulas como parte da sua jornada laboral. Estes descreveram como suas características pessoais, formação acadêmica, experiência profissional e os recursos do ambiente interferem no seu trabalho docente e que importância eles dão a suas funções de planejamento, desenvolvimento e avaliação 
da formação. Evidenciou-se que o seu trabalho é baseado em modelos que trazem das suas preconcepções docentes ou da relação com os seus pares. Seu alto grau de especialização técnica, traduzida em formação, é um elemento de motivação para realizar a docência. Discute-se que ser um bom professor com dedicação parcial e identificar-se com isto, contém algo de realização pessoal, mas também é consequência do modo de trabalhar, graças ao apoio de um grupo de trabalho, um departamento, procedimentos, valores e formação permanente.

Palavras chave: educação superior técnica profissional, formador da educação superior técnica profissional, papel docente.

\section{INTRODUCCIÓN}

\subsection{EDUCACIÓN SUPERIOR TÉCNICO PROFESIONAL EN CHILE}

Actualmente, alrededor del 50\% de la matrícula de la formación superior técnico profesional está contemplada por Institutos Técnicos Profesionales (IP) y Centros de Formación Técnica (CFT), donde el $60 \%$ de los estudiantes pertenece al sector más pobre de la población chilena $\left(\mathrm{CINDA}^{1}, 2008\right)$. Esta formación se valora muchísimo debido al aporte que puede hacer a la democratización del acceso a la enseñanza terciaria y a su posterior contribución a la formación de un capital humano especializado, de particular relevancia para la competitividad de la economía y para impulsar una creciente sofisticación del sistema productivo (Meller y Brunner, 2009; FUNDACIÓN CHILE, 2004; OIT, 2003). Este tipo de formación es una excelente alternativa para los estudiantes y su matrícula sigue en aumento porque:

- Es una opción a corto plazo para obtener un título específico, habilitante para el trabajo.

- La tasa de empleabilidad y retorno económico es alta, si el programa está bien definido en cuanto a las necesidades de la industria.

A pesar de la oferta existente, se evidencia que Chile no cuenta con un sistema de formación técnico profesional bien definido, imposibilitando las trayectorias progresivas entre los diferentes niveles de formación (secundaria y universitaria). Se verifica, entonces, que las principales dificultades tienen que ver con la deficiente reglamentación sobre la docencia en este sistema educativo (Meller y Brunner, 2009). Ahora bien, para que un sistema educativo de estas características funcione, su diseño y desarrollo ha de estar estrechamente relacionado y en concordancia con las distintas instancias educativas, el sector productivo de un país y en constante adaptación a sus demandas laborales (Marchesi, 2009). Esto se debe a que los programas académicos se construyen por competencias, siendo éstas las necesarias para cubrir un puesto de trabajo en el mundo laboral. De ahí la importancia de contar con gente especializada en los ámbitos educativos y de formación permanente, y con ofertas de calidad, suficientes y descentralizadas.

Por otro lado, un factor importante es que no existe un marco competencial definido en la industria de las tecnologías, que es el área de los profesionales de este estudio, acarreando problemáticas de currículo y de docencia en las instituciones educativas. Así, pues, las competencias profesionales que deben ser entregadas en los cursos académicos son construidas en función de la demanda profesional, siendo el mismo sector industrial

Centro Interuniversitario de Desarrollo (CINDA) del Grupo Operativo de Universidades Chilenas, MINEDUC, Chile. 
quien define y valida los conocimientos, habilidades y actitudes habilitantes para un puesto de trabajo. Asimismo, son los profesionales que trabajan en la industria los que se requieren para llevar a cabo el proceso formativo de los estudiantes. Ellos son los que entregan las características personales asociadas a su desarrollo y su entorno, y que se relacionan fundamentalmente con la capacidad laboral, medible y necesaria para realizar un trabajo eficazmente, es decir, producir los resultados deseados por la organización donde los trabajadores deben dar cuenta del logro de sus metas u objetivos de acuerdo a su rol (Fernández, 2007; Miranda, 2003; Boyatzis, 1982; McClelland, 1973).

\subsection{EL FORMADOR DE LA FORMACIÓN PROFESIONAL CHILENA: ROL Y PROFESIONALISMO DOCENTE}

Grollman \& Rauner (2007) identificaron varios perfiles docentes, destacándose los profesionales que trabajan en el sector productivo. Es así como, alrededor del 70\% de los formadores de la Educación Superior Técnico Profesional trabajan en jornada parcial o por horas (CSE, 2010; Castillo y Alzamora, 2009), lo que concuerda con lo requerido por los modelos educativos impartidos por este tipo de instituciones. Por tanto, estos formadores se caracterizan por ser profesionales vinculados a la industria, quienes transmiten la experiencia profesional y transfieren las competencias definidas en los planes de estudio. Así, es clave el valor agregado que ellos añaden, ya que ellos cuentan e importan en la mejora de la enseñanza (CINDA, 2008; Careaga, 2007).

El proceso de transferencia de experiencia de los formadores a los estudiantes no sólo se reduce a la transmisión de conocimientos técnicos a través de la práctica, sino que, además, existe un gran porcentaje de otras habilidades y actitudes que dan cuenta de una formación integral que les permite estar atentos a los avances tecnológicos y curriculares. Ciertamente, la enseñanza es un trabajo en que las emociones son fundamentales, ya que los formadores imponen de alguna forma su yo personal y profesional a la hora de realizar su tarea (Castillo y Alzamora, 2009; García, 2009; Day, 2006). Tal como lo señala Careaga: "tener estudiantes a su cargo y dar clases no significa ser docente" (2007: 2). Según la autora, la docencia no comprende cursar una carrera o cursarla una sola vez, sino que hacer docencia es un proceso que se adquiere a lo largo de la vida.

Ser profesor en los nuevos escenarios de vulnerabilidad social, surgimiento de nuevas configuraciones familiares e identidades juveniles, diferencias culturales, hacen que la profesión docente no se limite a tener personas sólo con un título académico y formación pedagógica. En concordancia con estos nuevos cambios, la labor docente debe realizarse en tres dimensiones: la del aprendizaje del estudiante, la de gestión educativa y la de políticas educativas (Murillo, González y Riso, 2009; Montero Lago, 2007). Y ésta será fuente de ingreso, poder y prestigio en la medida que las funciones que realicen en estos contextos sean específicas, concretas y delimitadas (Navío, 2006).

La tendencia de la educación en el último siglo es que el formador debe cumplir el rol de facilitador del aprendizaje de los alumnos, otorgándole cada vez mayor protagonismo al estudiante en su proceso de formación (Salas, 2005). Contrario a lo que se define conceptualmente, la realidad es otra. La experiencia nos indica que estos profesionales no cuentan con las herramientas necesarias para ser facilitadores, como lo exige el modelo educativo, sino que son más bien especialistas de un área en particular. En efecto, las instituciones educativas buscan profesionales expertos en alguna especialidad de sus 
programas curriculares, sustentando la primera condición del rol docente: ser transmisor de competencias y experiencia técnica. Incluso valorizan más a un docente certificado ${ }^{2}$ del que no lo es, potenciando la formación en estos ámbitos, sobre otras habilidades y competencias actitudinales. Por otro lado, la escasa valoración dada por los directivos, jefes y administrativos de estas instituciones a la comunicación entre los departamentos y los profesionales con dedicación parcial a la docencia, afecta en el fortalecimiento del vínculo afectivo que se deriva del sentimiento de pertenencia a una red. La poca cantidad de horas que pasan en la institución los limita a optar a otras actividades, generando situaciones de desmotivación e interrupciones de iniciativas de colaboración entre pares, con la institución y, en definitiva, en el contexto educacional (Knight, 2005).

El éxito de cualquier carrera profesional necesita del acompañamiento de factores relevantes relacionados con la gestión institucional (selección, inmersión inicial, perfeccionamiento inicial y continuo, política salarial, inspección, evaluaciones externas), su entorno sociocultural, desarrollo, identidad y valoración social (Pérez Gómez, 2010; Vaillant, 2007). Aún más, la proyección de ésta en el tiempo implica preparación, observación y reflexión en el entorno donde se está inmerso. La combinación de estos elementos permite la profesionalización de la carrera docente (Navío, 2005; VillegasReimser, 2003; Tejada, De la Torre y Barrios, 2000; Le Boterf, 1999).

Asimismo, la concepción que los profesionales tienen acerca de su propia actividad es esencial, dado que sólo la historia personal y una aproximación global a la individualidad de cada uno permite acceder a lo que significa ser formador, a cómo se entiende a sí mismo, al compromiso que mantiene con su profesión, a la satisfacción o insatisfacción que muestra su ánimo, a la idea acerca de cómo entiende su función social, a cuáles son los supuestos o teorías implícitas que sustentan su visión de los estudiantes y el valor de la educación (Gimeno, 2010; Silva, 2009). El proceso de identificación con el trabajo docente es una construcción del mismo profesional e incluye: el compromiso personal, la habilidad para aprender a enseñar, las creencias, los valores, los conocimientos sobre la materia que enseñan, las experiencias pasadas, así como la vulnerabilidad profesional, en cuanto interactúa en su contexto personal, profesional y situacional (Day \& Kington, 2011; Day, Kington, Stobart \& Sammons, 2006; Lasky, 2005).

\subsection{FACTORES QUE INFLUYEN EN LA PERCEPCIÓN QUE TIENEN LOS PROFESIONALES SOBRE SU TAREA DOCENTE}

Los profesionales cuya dedicación a la docencia es parcial, sitúan su trabajo técnico por sobre el docente durante su jornada de trabajo y ellos son quienes deciden en qué tarea creen desempeñarse mejor, participar o comprometerse en el tiempo. En consecuencia, se identifican con la tarea que está en primer lugar: su profesión técnica. De esto se deriva que la docencia suele no ser considerada como una actividad profesional que requiere principios técnicos, científicos, conceptuales, axiológicos, filosóficos e ideológicos, sino un oficio que se deriva del sello vocacional (Villarruel, 2012).

Knight (2005) revela que las razones que tienen estos profesionales para trabajar con dedicación parcial, están relacionadas con:

Certificado que cuenta con la garantía y pruebas suficientes de la experticia en alguna área técnica. 
- Tratar de alcanzar la estabilidad como formadores de la educación superior, ya que ven la enseñanza como un camino de oportunidades de aprendizaje, desarrollo y estímulo profesional, reconocimiento y prestigio.

- La necesidad de obtener dinero con urgencia.

- Prescindir de la obligación y compromiso con respecto a la comunidad de práctica profesional docente.

Estudios previos señalan que los principales factores que afectan al rol docente tienen relación con las emociones, la motivación, la satisfacción por el trabajo, el compromiso, la autoeficacia y las experiencias previas. Estos elementos determinan el comportamiento respecto con su trabajo, las formas, dirección, intensidad y duración del mismo, en la interacción con otros y en la reflexión de sus prácticas pedagógicas y de su desarrollo profesional (Ávalos, 2012; Canrinus, Helms, Beijaard, Buitink \& Hofman, 2011; Hildebrandt \& Eom, 2011; Day et al., 2006; Day, Elliot \& Kington, 2005; Latham \& Pinder, 2005; Zembylas, 2003; Hargreaves, 2001). De igual modo, estos factores influyen directamente con la imagen que él tiene de sí mismo, en su percepción y perspectiva futura, en sus creencias y valores, y en su bienestar emocional (Lasky, 2003).

También los mecanismos y estrategias de gestión docente que tienen las instituciones son fundamentales para convocar y mantener a los profesionales en su rol de formador. Estrategias de reclutamiento, inducción institucional y educativa, y perfeccionamiento docente son clave para controlar la deserción (Ávalos \& Aylwin, 2007; Velasco, 2007; Day et al., 2006, 2005; Lasky, 2005; Van Den Berg, 2002).

La motivación central de la investigación se sustenta en los formadores con dedicación parcial a la docencia en la Educación Superior Técnico Profesional. Se analizó cómo perciben su rol de formador, bajo los siguientes antecedentes:

- La desconsideración social existente de la formación superior técnica profesional con respecto a la formación tradicional universitaria.

- Las regulaciones contractuales que afectan la satisfacción, el compromiso y sobre todo la motivación por el trabajo docente.

- Las estrategias y mecanismos institucionales en el desarrollo de ambientes de colaboración, en los planes de perfeccionamiento y en el desarrollo docente.

Por tanto, los objetivos definidos para el estudio fueron:

- Indagar en los antecedentes personales, formación académica y experiencia profesional y ver cómo éstos influyen en sus apreciaciones sobre la docencia.

- Analizar en qué medida los recursos de su entorno empresarial y educacional son importantes.

- Examinar el nivel de relevancia que estos profesionales otorgan a sus labores docentes de planificación, desarrollo y evaluación de la formación que realizan.

\section{METODOLOGÍA}

El diseño de la investigación consideró la combinación de dos metodologías: una cuantitativa de tipo no experimental, y otra cualitativa para completar la información. No obstante, el presente artículo sólo considera el análisis de los resultados cuantitativos. 
Fue un estudio descriptivo, ya que a partir de la recogida de información dada por la muestra se pretendió describir las apreciaciones de los informantes en cuanto a los factores que afectan su labor, hasta el momento en que fueron recopilados los datos.

\subsection{MUESTRA}

La muestra consideró a profesionales técnicos e ingenieros de una institución de Educación Superior Técnico Profesional $(n=150)$. Finalmente, la muestra real fue $n=59$. Los participantes fueron aquellos que trabajan en la industria y en la docencia. Su jornada de trabajo contemplada para este estudio fue agrupada en: jornada diurna y vespertina (docentes que trabajan en ambas) y jornada diurna o vespertina (docentes que trabajan en una de las dos).

El tipo de muestreo fue no probabilístico e intencional. Es frecuente, en estos casos, utilizar como muestra a los individuos a los que se tiene fácil acceso, por derivar todos de una institución.

\subsection{INSTRUMENTOS}

Los instrumentos utilizados fueron de dos tipos: uno cuantitativo compuesto por un cuestionario, y otro cualitativo constituido por una entrevista. Este último sólo fue usado para complementar la información.

Por la distancia geográfica y diferencia horaria con los informantes claves, se procedió a elaborar el instrumento cuantitativo a través de una plataforma web, favoreciendo con ello la recogida de datos. Para su construcción, primeramente, se identificaron las variables de estudio a partir de la revisión contextual. Éste contempló dos partes, respectivamente:

\subsubsection{Variables relacionadas con aspectos generales}

- Personales: edad, sexo, sede a la que pertenece, carrera en que realiza clases, jornada, categoría docente y lugar de nacimiento.

- Formativos (contexto profesional y docente): profesión, tipo de carrera (titulación), certificaciones técnicas, cursos técnicos realizados en los últimos 5 años, grado académico y cursos de formación docente realizados en los últimos 5 años.

- Laborales (experiencia laboral y docente): cargos (puestos de trabajo en la industria) ocupados en los últimos 5 años, pertenencia a alguna comunidad tecnológica, pertenencia a alguna asociación profesional, cargos en docencia ocupados en los últimos 5 años y pertenencia a alguna comunidad educativa.

\subsubsection{Variables relacionadas con el perfil del docente (entorno y aspectos metodológicos)}

- Variables del entorno: características de desarrollo profesional, características sobre la labor docente, competencias actitudinales, características de satisfacción personal.

- Variables metodológicas: tareas de planificación, tareas de desarrollo y tareas de la evaluación de los cursos que imparten.

Las preguntas fueron con respuestas dicotómicas y categorizadas, y otras del tipo abiertas. En el caso de las preguntas de valoración se utilizó una escala tipo Likert. 
Para el análisis de los datos se realizó análisis estadístico e inferencial, mediante el uso del programa informático SPSS versión 20. En el primero, se efectuaron cálculos de frecuencia, media aritmética y desviación estándar, de manera de determinar la tendencia central y con el objetivo de identificar los intervalos en cada escala y hacer las respectivas representaciones gráficas para las variables. Para el análisis inferencial se usaron pruebas no paramétricas para las variables independientes.

\section{RESULTADOS DEL ESTUDIO}

Tal como se mencionó en la metodología, se exhibe sólo la caracterización y el análisis de los resultados derivados de la aplicación del cuestionario para los formadores de la Educación Superior Técnico Profesional.

Primero se hace una caracterización del grupo objeto de estudio en función de las variables relacionadas con aspectos generales (personales, formativos y laborales), para luego presentar los resultados de las variables relacionadas con el perfil del docente (entorno y aspectos metodológicos), y un análisis de los resultados de las mismas, de acuerdo a dos variables independientes: la edad y la jornada de trabajo. Se tomará como referencia el análisis de medias (M) y desviaciones estándar (SD) de cada una de las variables.

\subsection{CARACTERIZACIÓN DEL GRUPO DE ESTUDIO}

\subsubsection{Sexo y edad}

El sexo predominante es el masculino. Este fenómeno se relaciona con la tendencia de que mayoritariamente son los hombres los interesados en cursar carreras técnicas e ingeniería. La edad de los participantes fluctuó entre 21 y 63 años. Sin embargo, para el análisis posterior se agrupo la edad en 3 tramos (T1, T2, T3). El detalle se puede apreciar en la Tabla $\mathrm{N}^{\circ} 1$.

Tabla $N^{\circ}$ 1. Distribución de participantes por edad-sexo

\begin{tabular}{|c|c|c|c|c|}
\hline \multirow{2}{*}{ Edad } & \multicolumn{2}{|c|}{ Sexo } & \multirow{2}{*}{ Total } & Porcentaje \\
\cline { 2 - 5 } & Mujer & Hombre & 12 & $20,34 \%$ \\
\hline T1:Menores de 30 & 2 & 10 & 27 & $45,76 \%$ \\
\hline T2:Entre 30 y 39 & 2 & 25 & 20 & $33,90 \%$ \\
\hline T3:Mayores de 39 & 1 & 19 & 59 & $100 \%$ \\
\hline Total & 5 & 54 & & \\
\hline
\end{tabular}

Fuente: elaboración propia. 


\subsubsection{Jornada de trabajo versus edad}

La muestra se divide en dos proporciones con respecto a la jornada en la que realizan clases. Una categoría de trabajo (J1) diurna o vespertina con $61,02 \%$, y otra categoría de trabajo (J2) perteneciente a ambas jornadas: diurna y vespertina con $38,98 \%$. Se destaca que la mayor cantidad de profesionales pertenecen al T3 de edad (entre 30 y 39 años) y son de la categoría de trabajo (J1).

\subsubsection{Categoría docente en jornada}

Se evidenciaron las siguientes categorías docentes:

- Instructor: docente certificado con contrato indefinido.

- Adjunto: docente que además realiza tareas administrativas con contrato indefinido.

- Auxiliar definido: docente con contrato a plazo fijo.

- Auxiliar indefinido: docente con contrato indefinido.

En la Tabla $\mathrm{N}^{\circ} 2$ se puede observar que la mayor cantidad de participantes con contrato a plazo fijo se encuentra trabajando en una de las dos jornadas, lo que tiene sentido, debido a que para la mayoría de los formadores hacer docencia es sólo una pequeña parte de su jornada laboral:

Tabla $N^{\circ}$ 2. Distribución de participantes por categoría-jornada

\begin{tabular}{|l|c|c|c|c|}
\hline \multirow{2}{*}{ Categoría } & \multicolumn{2}{|c|}{ Jornada } & \multirow{2}{*}{ Total } & \multirow{2}{*}{ Porcentaje } \\
\cline { 2 - 3 } & J1 & J2 & & \\
\hline Instructor & 12 & 6 & 18 & $30,51 \%$ \\
\hline Adjunto & 3 & 4 & 7 & $11,86 \%$ \\
\hline Auxiliar Definido & 19 & 10 & 29 & $49,15 \%$ \\
\hline Auxiliar Indefinido & 2 & 3 & 5 & $8,47 \%$ \\
\hline Total & 36 & 23 & 59 & $100 \%$ \\
\hline
\end{tabular}

Fuente: elaboración propia.

\subsubsection{Formación profesional y técnica}

Los formadores se caracterizan por proceder de carreras técnicas, por sobre las carreras de profesorado o de una ingeniería de mayor nivel, como parte de su formación inicial. En efecto, la proporción de profesionales de ingenierías técnicas es de 66,1\%. Es así como los del tramo T2 de edad concentra la mayor distribución de este tipo, con un 53,84\%.

Con respecto a los grados académicos y certificaciones es posible observar en la Tabla $\mathrm{N}^{\circ} 3$ que el mayor porcentaje de participantes no tiene grado académico. Al contrario, 
encontramos que el mayor porcentaje de profesionales certificados son del T2 de edad $(48,38 \%)$ del total del certificados que cubre un 55,93\% del total de encuestados. Esto se debe a que la exigencia de la institución es contar con profesionales altamente especializados en alguna temática técnica más allá de los grados académicos (maestría o doctorado). Por otro lado, la formación de estos profesionales en aspectos metodológicos es baja. En los últimos 5 años sólo un 26,3\% dice haber realizado algún curso en estas materias, y la mayoría de éstos son obligatorios e impuestos por la institución.

Tabla $N^{\circ}$ 3. Distribución de participantes por grado académico-edad

\begin{tabular}{|l|c|c|c|c|c|}
\hline \multirow{2}{*}{ Grado Académico } & \multicolumn{3}{|c|}{ Edad } & \multirow{2}{*}{ Total } & \multirow{2}{*}{ Porcentaje } \\
\cline { 2 - 4 } & T1 & T2 & T3 & & \\
\hline Licenciado & 2 & 10 & 9 & 21 & $35,59 \%$ \\
\hline Máster & 2 & 7 & 2 & 11 & $18,64 \%$ \\
\hline Doctor & 0 & 0 & 0 & 0 & $0 \%$ \\
\hline No tiene & 8 & 10 & 9 & 27 & $45,76 \%$ \\
\hline Total & 12 & 27 & 20 & 59 & $100 \%$ \\
\hline
\end{tabular}

Fuente: elaboración propia.

\subsubsection{Experiencia laboral y docente}

Se pudo constatar que existe un alto número de profesionales $(54,24 \%)$ cuya experiencia en la industria es en cargo técnico. Otro aspecto a destacar es que en este grupo de profesionales la mayoría son del T2 de edad (entre 30 y 39 años). Por otro lado, a nivel de experiencia docente los encuestados dicen que en los últimos 5 años han realizado docencia en formación profesional (52,63\%), en formación universitaria y profesional (24,56\%), en capacitación a empresas y en formación profesional (14,04\%), y en las tres modalidades a la vez $(8,77 \%)$.

Finalmente, cabe señalar que estos profesionales evidencian una alta participación en comunidades tecnológicas $(64,41 \%)$, por sobre asociaciones profesionales $(18,64 \%)$ y comunidades académicas $(28,81 \%)$. El alto número de profesionales pertenecientes a una comunidad tecnológica se centra en el T2 de edad.

\subsection{RESULTADOS Y ANÁLISIS DE LAS VARIABLES RELACIONADAS CON EL PERFIL DOCENTE Y QUE INTERVIENEN EN LAS PERCEPCIONES DE LOS FORMADORES}

\subsection{1. $\quad$ Percepciones de los formadores}

\subsubsection{Características de desarrollo profesional}

Se evidencia una alta valoración de la especialidad $(\mathrm{M}=4,15$ y $\mathrm{SD}=1,15)$ y de la vinculación con la industria $(\mathrm{M}=4,05$ y $\mathrm{SD}=1,14)$ como características fundamentales para un desarrollo 
Estudios Pedagógicos XLI, $\mathrm{N}^{\circ}$ 1: 45-61, 2015

FORMADOR DE LA EDUCACIÓN SUPERIOR TÉCNICO PROFESIONAL: PERCEPCIONES SOBRE SU TRABAJO

DOCENTE

docente. Esto coincide con la premisa institucional: contar con profesionales especialistas (certificados) y con alta vinculación con el sector productivo.

\subsubsection{Características de la labor docente}

Las características de la labor docente seleccionadas como importantes fueron ${ }^{3}$ : generar valor en los alumnos $(\mathrm{M}=1,23$ y $\mathrm{SD}=0,42)$ y formar profesionales calificados $(\mathrm{M}=1,25 \mathrm{y}$ $\mathrm{SD}=0,43)$, más que ser un aporte a la sociedad $(\mathrm{M}=1,39$ y $\mathrm{SD}=0,42)$ y ser referentes para el alumno $(\mathrm{M}=1,33$ y $\mathrm{SD}=0,47)$, considerando que estos dos últimos son objetivos docentes importantes difundidos por la institución.

\subsubsection{Competencias actitudinales}

La competencia actitudinal más valorada es la responsabilidad en el trabajo $(M=4,63$ y $\mathrm{SD}=0,92)$. En cambio, las competencias menos apreciadas resultaron ser: liderazgo $(\mathrm{M}=4,32$ y $\mathrm{SD}=1,18)$, exigencia $(\mathrm{M}=4,09$ y $\mathrm{SD}=0,96)$ y sensibilidad social $(\mathrm{M}=4,05$ y $\mathrm{SD}=1,14)$. Mientras que, para la institución, éstas últimas son necesarias y habilitantes para desarrollar la labor docente, ya que les permite comprender y situar mejor su tarea.

\subsubsection{Características de satisfacción personal}

La instrucción permanente en el ámbito metodológico $(\mathrm{M}=3,80$ y $\mathrm{SD}=1,14)$, el grado de pertenencia con la institución $(\mathrm{M}=3,75$ y $\mathrm{SD}=1,29)$ y los recursos disponibles $(\mathrm{M}=$ $3,72$ y $\mathrm{SD}=1,29)$ son las características más apreciadas. No obstante, estos resultados son paradójicos considerando la baja formación de los profesionales en estas temáticas.

\subsubsection{Tareas metodológicas}

En la planificación de la formación las tareas más importantes son: determinar los objetivos del curso $(\mathrm{M}=4,40$ y $\mathrm{SD}=0,90)$ y la evaluación de contenidos $(\mathrm{M}=4,23$ y $\mathrm{SD}=0,91)$. En contraste, la valoración más baja la tiene la tarea de identificar al estudiante con el cual interactuarán ( $\mathrm{M}=3,96$ y $\mathrm{SD}=1,02)$, considerada esencial en el contexto institucional para proyectar cómo se desarrollará el curso (por ejemplo, suplir deficiencias de los estudiantes).

En el desarrollo de la formación, la comunicación efectiva $(\mathrm{M}=4,5$ y $\mathrm{SD}=0,95)$ y el manejo de grupo $(\mathrm{M}=4,40$ y $\mathrm{SD}=0,94)$ son competencias fundamentales para el éxito del curso, no así el manejo de diferencias sociales $(\mathrm{M}=4,1$ y $\mathrm{SD}=1,02)$ y la tolerancia a la presión $(\mathrm{M}=4,19$ y $\mathrm{SD}=1,01)$, siendo estas últimas importantes en el contexto social y cultural donde se desenvuelve la institución.

En la evaluación de la formación, el análisis de resultados $(\mathrm{M}=4,23$ y $\mathrm{SD}=1,04)$ y la revisión y entrega de los mismos $(\mathrm{M}=4,21$ y $\mathrm{SD}=1,07)$ son las tareas más significativas. Por otro lado, se evidenció la poca preocupación en la definición y el diseño de los instrumentos de evaluación ( $\mathrm{M}=4,14$ y SD=0,97). Esto se explica por la incipiente implementación de la fase de evaluación del modelo educativo en la institución.

Se consideraron como valores de selección a $1=$ característica seleccionada y $2=$ característica no seleccionada. 


\subsection{2. $\quad$ Análisis de las percepciones de acuerdo a las variables independientes}

\subsubsection{En función de la variable edad}

En el caso de las características de desarrollo profesional no se encontraron diferencias significativas. Indistintamente de la edad, los formadores valoran las certificaciones en la línea de especialidad técnica, lo que tiene coherencia con lo expuesto en el marco referencial. Otro punto destacable es que estos profesionales consideran menos importante tener una experiencia docente previa, lo que concuerda con hallazgos previos que dicen que no es necesario ser "docente" para transferir los conocimientos técnicos.

Respecto de las características de satisfacción personal es posible observar diferencias significativas en la estabilidad laboral (Tabla $\mathrm{N}^{\circ} 4$ ). Esto se debe a la necesidad de los profesionales de mayor edad de permanecer en la docencia, versus los profesionales más jóvenes que están en pleno desarrollo y experiencia profesional en su línea de especialidad técnica, siendo esta última la que les brinda mejor estabilidad laboral.

Tabla $N^{\circ}$ 4. Medias de las percepciones docentes según edad referidas a características de satisfacción personal

\begin{tabular}{|c|c|c|c|c|c|c|}
\hline \multirow{2}{*}{$\mathbf{N}^{\circ}$} & \multirow{2}{*}{ Satisfacción personal del entorno } & \multicolumn{3}{|c|}{ Edad } & \multirow{2}{*}{ Sig. ${ }^{* 4}$} & \multirow{2}{*}{ Diferencias } \\
\hline & & T1 & $\mathbf{T 2}$ & T3 & & \\
\hline 1 & Valor/Hora docente. & 3,27 & 3,26 & 3,63 & - & - \\
\hline 2 & Estabilidad laboral (tipo de contrato). & 2,47 & 3 & 3,79 & 0,035 & $3>2>1$ \\
\hline 3 & Grado de pertenencia con la institución. & 3,4 & 3,57 & 4,26 & - & - \\
\hline 4 & Apoyo de las autoridades institucionales. & 3,93 & 3,35 & 3,63 & - & - \\
\hline 5 & Trabajo colaborativo entre docentes pares. & 3,13 & 3,43 & 3,63 & - & - \\
\hline 6 & $\begin{array}{l}\text { Recursos disponibles para impartir sus clases } \\
\text { (infraestructura, salas, laboratorios, servicios, otros). }\end{array}$ & 3,6 & 3,48 & 4,11 & - & - \\
\hline 7 & Exigencias del modelo educativo. & 3,67 & 3,43 & 3,95 & - & - \\
\hline 8 & Exigencias administrativas. & 3,27 & 3,39 & 3,74 & - & - \\
\hline 9 & Instrucción permanente disciplinaria. & 3,47 & 3,17 & 3,74 & - & - \\
\hline 10 & Instrucción permanente metodológica. & 3,4 & 3,65 & 4,26 & - & - \\
\hline
\end{tabular}

Fuente: elaboración propia.

En el caso de las tareas metodológicas, concretamente en relación con la función de planificación de la formación, se evidencia una diferencia para la tarea de definición de las estructuras de evaluación (Tabla $\mathrm{N}^{\circ}$ 5). En efecto, se puede observar que los grupos de mayor y menor edad son los que aprecian mayormente esta tarea, no así los de mediana edad. Esta situación se debe a la estructura de instrucción de inicio y de formación previa que tienen los formadores y los modelos que aprendieron en su etapa de estudiantes.

\footnotetext{
* Nivel de significación $<0,05$.
} 
Tabla $N^{\circ}$ 5. Medias de las percepciones docentes según edad referidas a las tareas de planificación de la formación

\begin{tabular}{|c|c|c|c|c|c|c|}
\hline \multirow{2}{*}{$\mathbf{N}^{\circ}$} & \multirow{2}{*}{ Tareas de planificación de la formación } & \multicolumn{3}{|c|}{ Edad } & \multirow[t]{2}{*}{ Sig. ${ }^{* 5}$} & \multirow[t]{2}{*}{ Diferencias } \\
\hline & & T1 & $\mathbf{T} 2$ & $\mathbf{T 3}$ & & \\
\hline 1 & $\begin{array}{l}\text { Análisis de la formación a impartir. Revisión } \\
\text { Programa Instruccional de la Asignatura (PIA). }\end{array}$ & 4,07 & 4,09 & 4,42 & - & - \\
\hline 2 & $\begin{array}{l}\text { Análisis de las características de los estudiantes } \\
\text { que recibirán formación (curso, nivel). }\end{array}$ & 3,8 & 3,83 & 4,26 & - & - \\
\hline 3 & $\begin{array}{l}\text { Definición de los objetivos del curso, es decir, lo } \\
\text { que se desea lograr con la formación de acuerdo a } \\
\text { las competencias declaradas en el PIA. }\end{array}$ & 4,4 & 4,17 & 4,63 & - & - \\
\hline 4 & $\begin{array}{l}\text { Identificación y organización de las competencias: } \\
\text { competencias, habilidades y actitudes a impartir. }\end{array}$ & 4,33 & 3,87 & 4,42 & - & - \\
\hline 5 & $\begin{array}{l}\text { Diseño de estrategias metodológicas. Revisión de } \\
\text { Programa Secuencial de Asignatura (PSA) y } \\
\text { material de apoyo. }\end{array}$ & 4 & 4 & 4,32 & - & - \\
\hline 6 & $\begin{array}{l}\text { Definición estructura de evaluación. Identificación } \\
\text { de instrumentos con los que se medirán las } \\
\text { competencias y los porcentajes de cada uno. }\end{array}$ & 4,33 & 3,87 & 4,58 & 0,033 & $3>1>2$ \\
\hline
\end{tabular}

Fuente: elaboración propia.

En cuanto a las tareas asociadas al desarrollo de la formación es destacable mencionar que, aunque no se aprecian diferencias significativas, los encuestados consideran que la comunicación efectiva y el manejo de grupo son tareas esenciales. Estos resultados concuerdan con las competencias profesionales con las que deben contar estos docentes.

\subsubsection{En función de la variable jornada de trabajo}

Respecto de las características de la labor docente se evidencia que los profesionales que trabajan en alguna de las jornadas (Diurna/Vespertina) creen ser referentes para el alumno y transferir conocimientos técnicos y experiencia práctica. Este fenómeno se da por su condición de docente con jornada parcial y su alta vinculación con la industria, a diferencia de los que trabajan en ambas jornadas, cuya relación con el mundo empresarial es menor.

En el caso de las características de desarrollo profesional es posible constatar una diferencia significativa (Tabla $\mathrm{N}^{\circ}$ 6) en la valoración dada a la experiencia docente previa. Los profesionales del grupo J1 (diurna o vespertina) dan mayor importancia a este ítem, y esto se sustenta fundamentalmente por la variedad de perfiles de alumnos con los que deben tratar, y la mayor cantidad de horas dedicadas a la docencia.

* Nivel de significación $<0,05$. 
Tabla $N^{\circ}$ 6. Medias de las percepciones docentes según jornada referidas a características de desarrollo profesional

\begin{tabular}{|c|l|c|c|c|c|}
\hline \multicolumn{2}{|c|}{ Características desarrollo profesional } & \multicolumn{2}{|c|}{ Jornada } & \multirow{2}{*}{ Sig. ${ }^{* 6}$} & \multirow{2}{*}{ Diferencias } \\
\cline { 3 - 6 } & Título profesional y grados académicos. & 3,96 & 4 & - & \\
\hline 2 & Formación docente previa. & 3,79 & 3,82 & - & - \\
\hline 3 & Formación pedagógica permanente. & 4,04 & 3,81 & - & - \\
\hline 4 & Experiencia docente previa. & 3,85 & 3,31 & 0,02 & J2>J1 \\
\hline 5 & Experiencia en la línea de experticia en la industria (años). & 4,21 & 4,05 & - & - \\
\hline 6 & Certificaciones en la línea de especialidad. & 3,8 & 3,88 & - & - \\
\hline 7 & Grado de vinculación con la industria. & 4,08 & 4 & - & - \\
\hline
\end{tabular}

Fuente: elaboración propia.

Asimismo, en las características de satisfacción personal se puede observar que los formadores del grupo 1 consideran relevante la estabilidad laboral o tipo de contrato (Tabla $\mathrm{N}^{\circ}$ 7). Por ende, al tener más horas en formación, ellos ven la docencia como una alternativa de carrera profesional, a diferencia del grupo 2 que pondera su labor en la industria como su principal fuente de ingreso.

Tabla $N^{\circ}$ 7. Medias de las percepciones de las características de satisfacción personal por jornada docente

\begin{tabular}{|r|l|c|c|c|c|}
\hline \multicolumn{2}{|c|}{ Satisfacción del entorno } & \multicolumn{2}{c|}{ Jornada } & \multirow{2}{*}{ Sig.* } & \multirow{2}{*}{ Diferencias } \\
\cline { 2 - 4 } 1 & Valor/Hora docente. & 3,35 & 3,43 & - & - \\
\hline 2 & Estabilidad laboral (tipo de contrato). & 3,24 & 2,96 & 0,034 & J2>J1 \\
\hline 3 & Grado de pertenencia con la institución. & 3,65 & 3,91 & - & - \\
\hline 4 & Apoyo de las autoridades institucionales. & 3,65 & 3,52 & - & \\
\hline 5 & Trabajo colaborativo entre docentes pares. & 3,41 & 3,43 & - & \\
\hline 6 & $\begin{array}{l}\text { Recursos disponibles para impartir sus clases } \\
\text { (infraestructura, salas, laboratorios, servicios, otros) }\end{array}$ & 3,85 & 3,52 & - & \\
\hline 7 & Exigencias del modelo educativo. & 3,59 & 3,78 & - & - \\
\hline 8 & Exigencias administrativas. & 3,62 & 3,26 & - & - \\
\hline 9 & Exigencias administrativas. & 3,5 & 3,35 & - & - \\
\hline 10 & Instrucción permanente metodológica. & 3,91 & 3,61 & - & - \\
\hline
\end{tabular}

Fuente: elaboración propia.

\footnotetext{
$6 \quad *$ Nivel de significación $<0,05$
} 
Por último, en cuanto a las tareas metodológicas de planificación de la formación, los formadores del grupo 1 priorizan la definición de objetivos del curso y análisis del mismo. En cambio, los formadores del grupo 2 (ambas jornadas) destacan la importancia de la revisión del programa instruccional de la asignatura. Estos contrastes radican fundamentalmente en que estos últimos, al tener menos horas de dedicación a la docencia, afirman la necesidad de apoyo e inducción en el desarrollo y el análisis de la formación que deben entregar.

\section{DISCUSIÓN}

El presente estudio nos revela que estos profesionales se caracterizan por ser principalmente personas de sexo masculino, de edad media (30 a 39 años), con un alto nivel de formación y experiencia técnica por sobre la pedagógica. Se destaca, además, que la gran parte de los profesionales con dedicación parcial trabajan en la industria, en cargos técnicos, donde experimentan a nivel productivo sus competencias profesionales y alto desarrollo en su línea de especialidad técnica. En efecto, estas características hacen que muchos de ellos, con independencia de lo que esperan y de lo que estén cualificados para hacer, tienen pocas perspectivas de desarrollar un carrera profesional docente (Knight, 2005). No obstante, la vasta experiencia de estos profesionales en el sector productivo y su tendencia a la especialización es fundamental para realizar formación en modelos educativos basados en competencias. En primer lugar, la vinculación, experiencia y especialización son requisitos de entrada para la formación profesional y, en segundo lugar, estos elementos son facilitadores para realizar la enseñanza de los conocimientos, habilidades y actitudes definidos en los programas académicos. Entonces, es concordante que los resultados evidencien una alta apreciación a la vinculación con la industria y a la especialización como características de desarrollo profesional.

De lo anterior se deriva que la especialización es una oportunidad de formación en los contextos educativos para estos formadores. Justamente, un factor clave presente en estudios previos señala que la capacitación y el aprendizaje continuo son elementos que influyen en la motivación que ellos tienen por el trabajo docente. Kirpal \& Witting (2009) destacaron que, en general, los profesionales que se dedican a la docencia en tiempo parcial gastan más de 8 horas al mes en capacitación en alguna línea de especialidad técnica. Tanto es así que este estudio constató que ellos tienen una actitud mucho más positiva hacia su labor docente, ya que ven este aprendizaje como beneficioso para su desarrollo profesional. Efectivamente, los aciertos de dicho estudio validan las apreciaciones que tienen los encuestados en cuanto a su tarea docente y, por otro lado, solicitan a la institución el desarrollo constante de estrategias de formación continua en estos aspectos.

Sin embargo, la insistente tendencia a la especialización olvida desarrollar otras habilidades que son importantes y que el docente debe adquirir para llevar a cabo su rol de facilitador. Es por ello que se postula que estos profesionales, más que ser facilitadores del aprendizaje en contextos de competencias, son especialistas en algún área técnica. Esto se relaciona con las percepciones que tienen los encuestados, que evidencian la falta de habilidades y actitudes necesarias para lograr el éxito en el desarrollo de la clase. Se suma, además, la poca formación en aspectos metodológicos que, si bien la mayoría de las percepciones vertidas en este estudio denota que no son necesarias, la realidad es otra. En 
efecto, las propias instituciones de educación debieran hacerse cargo de esta deficiencia, considerando que su modelo educativo requiere profesionales con este perfil.

Otro elemento destacable es el compromiso del docente con su labor. Se evidenció en los resultados de las encuestas la poca valoración dada al aporte a la sociedad que se puede hacer formando capital humano especializado. Esto último se valida con lo señalado por Ávalos, Cavada, Pardo y Sotomayor (2010), a saber, que los profesores conciben su misión formativa de un modo más amplio, en la medida en que las autoridades educativas privilegian una mirada enfocada sólo en los resultados por encima de las definiciones institucionales.

En lo que respecta a las percepciones sobre la labor docente, se coincide con lo expuesto por Ávalos (2012), quien señala que los formadores definen sus tareas que les son propias de manera que entienden sus relaciones con otros que hacen lo mismo, o bien de modelos que traen de sus preconcepciones cuando eran estudiantes. De lo anterior se deduce que la idea de ser un buen formador con dedicación parcial tiene algo de logro personal, también es un resultado del modo de operar gracias al apoyo de un grupo de trabajo, valores y procedimientos docentes (Tait, 2002). Por ello las estrategias para mejorar las prácticas docentes deben formularse en consonancia con los deseos, motivaciones y aspiraciones en conjunto con la comunidad académica.

Sería un gran avance que existan políticas educativas que consiguiesen regular la gestión docente de este tipo de instituciones. Más aún, si se tomasen en consideración las necesidades de estos profesionales y las incorporasen dentro de su gestión docente, esto optimizaría el entorno del formador $y$, a su vez, la calidad de enseñanza entregada en sus programas académicos.

Ciertamente, las limitaciones del estudio tienen relación con la muestra, al ser intencional e institucional, pudiendo no ser representativa y afectar en los posibles hallazgos. Además, al ser un estudio no experimental, se suma una limitación temporal, es decir, imposibilita profundizar en los cambios de las percepciones que podrían tener en un periodo de tiempo mayor. En consecuencia, los resultados aquí expuestos son sólo aproximaciones a las apreciaciones que estos formadores tienen sobre su labor y que se pueden tomar como referencia para otros estudios en contextos similares.

Para concluir, a partir de esta investigación es posible realizar aportaciones a futuros estudios relacionados. Se podría profundizar en los factores que afectan las percepciones que tiene este tipo de profesionales con respecto a su tarea docente en los diferentes contextos de acción, identificar en qué y cómo afectan en su desarrollo profesional docente, y asimismo, estas aproximaciones permitirían a las instituciones comenzar a reflexionar sobre lo que sus formadores necesitan para cumplir con éxito su tarea docente.

\section{REFERENCIAS BIBLIOGRÁFICAS}

Ávalos, B. (2012). Cómo ven su identidad los docentes chilenos. Revista Perspectiva Educacional, vol.51 (1), 77-95.

, Cavada, P., Pardo, M. y Sotomayor, C. (2010). La profesión docente: Temas y discusiones en la literatura internacional. Estudios Pedagógicos, vol.36 (1), 235-263.

\& Aylwin, P. (2007). How Young Teachers Experience their Professional Work in Chile. Teaching and Teacher Education, vol.23 (4), 515-528. 
Boyatzis, R. (1982). The Competent Manager: A Model for Effective Performance. New York: John Wiley \& Sons.

Canrinus, E., Helms, M., Beijaard, D., Buitink, J. A. \& Hofman, A. (2011). Profiling Teachers' Sense of Professional Identity. Educational Studies, vol.37 (5), 593-608.

Careaga, A. (2007). El desafío docente. Recuperado el 20 de diciembre de 2012 desde http:// www.medfamco.fmed.edu.uy/Archivos/pregrado/Ciclo_Introductorio/Materiales/Ser_docente.pdf

Castillo, D. y Alzamora, M. (2009). Situación de la práctica docente en la educación técnica superior. Recuperado el 20 de diciembre de 2012 desde http://www.revistaakademeia.cl/wp/wpcontent/uploads/2011/01/Situaci\%C3\%B3n-de-la-pr\%C3\%A1ctica-docente.pdf

CINDA (2008). Diseño curricular basado en competencias y aseguramiento de la calidad en la educación superior. Recuperado el 20 de diciembre de 2012 desde http://aula.virtual.ucv.cl/aula virtual/cinda/cdlibros/39-Dise\%C3\%B1o\%20Curricular\%20Basado\%20en\%20Competencias\%20 y $\% 20$ Aseguramiento $\% 20 \mathrm{de} \% 201 \mathrm{a} \% 20 \mathrm{Calidad} \% 20 \mathrm{en} \% 201 \mathrm{a} \% 20$ Educaci $\% \mathrm{C} 3 \% \mathrm{~B} 3 \mathrm{n} \% 20$ Superior/ Dise $\%$ C3\%B1o\%20Curricular\%20Basado\%20en\%20Competencias\%20y\%20Aseguramiento\%20 de\%20la\%20Calidad.pdf

CSE (2010). Índices docentes 2010. Recuperado el 6 de diciembre de 2012 desde http://www. cned.cl/public/secciones/seccionestadisticas/doc/Estadisticas2010/cse_a_f6c1_2009.pdf

Day, C. \& Kington, A. (2011). Identity, Well-being and Effectiveness: The Emotional Contexts of Teaching. Pedagogy, Culture \& Society, vol.16 (1), 7-23.

(2006). Pasión por enseñar: La identidad personal y profesional del docente y sus valores. Madrid: Narcea.

, Kington, A., Stobart, G. \& Sammons, P. (2006). The Personal and Professional Selves of

Teachers: Stable and Unstable Identities. British Educational Research Journal, vol.32 (4), 601-616. ., Elliot, B. \& Kington, A. (2005). Reform, Standards and Teacher Identity: Challenges of Sustaining Commitment. Teaching and Teacher Education, vol.21 (5), 563-577.

Fernández, B. (2007). Competencias laborales y de empleabilidad en la educación vocacional. Calidad en la Educación, (27), 36-51.

FUNDACIÓN CHILE (2004). Competencias laborales Chile 1999 - 2004. Recuperado el 20 de diciembre de 2012 desde http://www.oei.es/etp/competencias_laborales_chile1999_2004.pdf

García, C. (2009). Formalidad e informalidad en el proceso de aprender a enseñar. Revista de Educación, (350), 31-55.

Gimeno, S. J. G. (2010). La carrera profesional para el profesorado. Revista Interuniversitaria de Formación de Profesorado, vol.24 (2), 243-260.

Grollmann, P. \& Rauner, F. 2007. International Perspectives on Teachers and Trainers in Technical and Vocational Education. Dordrecht, Netherlands: Springer.

Hargreaves, A. (2001). Emotional Geographies of Teaching. Teachers College Record, vol.103 (6), 1056-1080.

Hildebrandt, S. \& Eom, M. (2011). Teacher Professionalization: Motivational Factors and the Influence of Age. Teaching and Teacher Education, vol.27 (2), 416-423.

Kirpal, S. \& Witting, W. (2009). Training Practitioners in Europe: Perspectives on their Work, Qualification, and Continuing Learning. Retrieved on January 9, 2013 from http://elib.suub.unibremen.de/ip/docs/00010607.pdf

Knight, P. (2005). El profesorado de educación superior: Formación para la excelencia. Madrid: Narcea.

Lasky, S. (2005). A Sociocultural Approach to Understanding Teacher Identity, Agency and Professional Vulnerability in a Context of Secondary School Reform. Teaching and Teacher Education, vol.21 (8), 899-916.

(2003). Teacher Professional Vulnerability in a Context of Standards based Reforms. Annual Meeting of the American Educational Research Association. Chicago, IL, United States.

Latham, G. \& Pinder, C. (2005). Work Motivation Theory and Research at the Dawn of the 
Twenty-first Century. Annual Review of Psychology, (56), 485-516.

Le Boterf, G. (1999). Compétence et navigation professionnelle. Paris: Editions d'Organisation.

Marchesi, Á. (2009). Preámbulo. Retos actuales de la educación técnico-profesional. Metas educativas 2021. Recuperado el 3 de enero de 2013 desde http://www.oei.es/metas2021/ETP.pdf

McClelland, D. (1973). Testing for Competence rather than for Intelligence. American Psychologist, vol.28 (1), 1-14.

Meller, P. y Brunner, J. (2009). Educación técnico profesional y mercado laboral en Chile: Un reader. Recuperado el 3 de enero de 2013 desde http://www.refugioenchile.cl/data/archivos/ archivo_2011_08_22_16_54_27.pdf

Miranda, M. (2003). Transformación de la educación media técnico-profesional. En C. Cox (Ed.), Políticas educacionales en el cambio de siglo (pp. 375-417). Santiago de Chile: Editorial Universitaria.

Montero Lago, P. (2007). Desafíos para la profesionalización del nuevo rol docente universitario. Ensaio: Avaliação e Políticas Públicas em Educação, vol.15 (56), 341-350. Recuperado el 3 de enero de 2013 desde http://www.scielo.br/scielo.php?script=sci_arttext\&pid=S010440362007000300003\&lng=en\&tlng=es. 10.1590/S0104-40362007000300003

Murillo, J., González, V. y Riso, H. (2006). Algunos conceptos y tendencias sobre carrera profesional docente. Recuperado el 9 de enero de 2013 desde http://images.buenadocencia. multiply.multiplycontent.com/attachment/0/TAG5kQooCqAAAHpJkTw1/DocenciaNo29-Carrera Profesional Docente.pdf?nmid=339857900

Navío, A. (2006). La formación de los profesionales de la formación para el trabajo: Algunos dilemas y algunas respuestas. Revista Educar, (38), 63-79. . (2005). Las competencias profesionales del formador: Una visión desde la formación continua. Barcelona: Octaedro.

OIT (2003). Aprender y formarse para trabajar en la sociedad del conocimiento. Recuperado el 9 de enero de 2013 desde http://www.oitcinterfor.org/sites/default/files/file_publicacion/rep-iv-1.pdf

Pérez Gómez, Á. I. (2010). Nuevas exigencias y escenarios para la profesión docente en la era de la información y de la incertidumbre. Revista Interuniversitaria de Formación de Profesorado, vol.24 (2), 17-36.

Salas, W. (2005). Formación por competencias en educación superior. Una aproximación conceptual a propósito del caso colombiano. Revista Iberoamericana de Educación, vol.36 (9), 1-10.

Silva, P. (2009). La profesión docente y la mejora de la calidad educativa. Nuevos retos de la profesión docente. II Seminario Internacional RELFIDO (pp. 9-11). Barcelona: Universidad de Barcelona.

Tait, J. (2002). From Competence to Excellence: A Systems View of Staff Development for Parttime Tutors at-a-distance. Open Learning, vol.17 (2), 153-166.

Tejada, J., De la Torre, S. y Barrios, O. (2000). Estrategias didácticas innovadoras: Recursos para la formación y el cambio. Barcelona: Octaedro.

Vaillant, D. (2007). Mejorando la formación y el desarrollo profesional docente en Latinoamérica. Revista Pensamiento Educativo, vol.41 (2), 207-222.

Van Den Berg, R. (2002). Teachers' Meanings regarding Educational Practice. Review of Educational Research, vol.72 (4), 577-625.

Velasco, C. (2007). Educación técnica y formación profesional en América Latina y el Caribe. Recuperado el 8 de enero de 2013 desde http://unesdoc.unesco.org/images/0016/001611/161174s.pdf

Villarruel, M. (2012). Identidad docente y exigencia académica: Encuentros y desencuentros con la realidad social. Perspectiva Educacional, vol.51 (1), 29-44.

Villegas-Remser, E. (2003). Teacher Professional Development: An International Review of the Literature. Retrieved on December 19, 2012 from http://www.iiep.unesco.org/fileadmin/user_ upload/Research_Challenges_and_Trends/133010e.pdf

Zembylas, M. (2003). Emotions and Teacher Identity: A Post-structural Perspective. Teachers and Teaching: Theory and Practice, vol.9 (3), 213-238. 
